\title{
Sharp dimension estimates of the attractor of the damped 2D Euler-Bardina equations
}

\author{
Alexei Ilyin \\ Sergey Zelik \\ To Ari Laptev on the occasion of his 70th birthday
}

\begin{abstract}
We prove existence of the global attractor of the damped and driven 2D Euler-Bardina equations on the torus and give an explicit two-sided estimate of its dimension that is sharp as $\alpha \rightarrow 0^{+}$.
\end{abstract}

Mathematics Subject Classification 2020. 35B40, 35B41, 37L30, 35Q31.

Keywords. Damped Euler-Bardina equations, $\alpha$ models, attractors, dimension estimates

\section{Introduction}

The Navier-Stokes system remains over the last decades in the focus of the theory of infinite dimensional dynamical systems (see, for example, $[2,12,22,28,31]$ and the references therein). For a system defined on a bounded two-dimensional domain it was shown that the corresponding global attractor has finite fractal dimension. The idea to use the Lieb-Thirring inequalities [24] for orthonormal families played an essential role in deriving physically relevant upper bounds for the dimension. Furthermore, the upper bounds in case of the torus $\mathbb{T}^{2}$ are sharp up to a logarithmic correction [25].

Another model in incompressible hydrodynamics more recently studied from the point of view of attractors is the two-dimensional damped Euler system

$$
\left\{\begin{array}{l}
\partial_{t} u+\left(u, \nabla_{x}\right) u+\gamma u+\nabla_{x} p=g \\
\operatorname{div} u=0, \quad u(0)=u_{0} .
\end{array}\right.
$$

A. Ilyin: Keldysh Institute of Applied Mathematics, Moscow, Russia; email: ilyin@keldysh.ru

S.Zelik: Keldysh Institute of Applied Mathematics, Moscow, Russia, School of Mathematics and Statistics, Lanzhou University, China and University of Surrey, Department of Mathematics, Guildford, GU2 7XH, United Kingdom; email: s.zelik@ surrey.ac.uk 
The linear damping term $\gamma u$ here makes the system dissipative and is important in various geophysical models [27]. The system is studied either on a $2 \mathrm{~d}$ manifold (torus, sphere) or in a bounded $2 \mathrm{~d}$ domain with stress-free boundary conditions. The natural phase space here is $H^{1}$ where it easy to prove the existence of a solution of class $L^{\infty}\left(0, T, H^{1}\right)$ and dissipativity. However, the solution in this class is not known to be unique.

A weak attractor and a weak trajectory attractor for (1.1) were constructed in [15] and [8], respectively. It was then shown in [9] and [10] that these attractors are, in fact, compact in $H^{1}$ and the attraction holds in the norm of $H^{1}$.

Closely related to (1.1) is its Navier-Stokes perturbation

$$
\left\{\begin{array}{l}
\partial_{t} u+\left(u, \nabla_{x}\right) u+\nabla_{x} p+\gamma u=v \Delta_{x} u+g, \\
\operatorname{div} u=0, \quad u(0)=u_{0}, x \in \mathbb{T}^{2}
\end{array}\right.
$$

which is studied in the vanishing viscosity limit $v \rightarrow 0$. It is proved in [9] that the attractors $\mathscr{A}_{v}$ of (1.2) tend in $H^{1}$ to the attractor $\mathscr{A}$ of (1.1), and, furthermore [18], their fractal dimension satisfies an order-sharp (as $v \rightarrow 0^{+}$) two sided estimate

$$
1.5 \cdot 10^{-6} \frac{\|\operatorname{curl} g\|_{L^{2}}^{2}}{v \gamma^{3}} \leq \operatorname{dim} \mathscr{A}_{v} \leq \frac{3 \pi}{128} \frac{\|\operatorname{curl} g\|_{L^{2}}^{2}}{v \gamma^{3}}
$$

where the left-hand side estimate holds for a specially chosen Kolmogorov forcing, and in the right-hand side we used the recent estimate of the Lieb-Thirring constant on the torus [16]. This indicates (or at least suggests) that the problem of estimating the dimension of the attractor $\mathscr{A}$ of (1.1) is difficult and the attractor may well be infinite dimensional.

In this work we use a different approximation of (1.1), namely, the so-called inviscid damped Euler-Bardina model (see $[3,4,21]$ and the references therein)

$$
\left\{\begin{array}{l}
\partial_{t} u+\left(\bar{u}, \nabla_{x}\right) \bar{u}+\gamma u+\nabla_{x} p=g, \\
\operatorname{div} u=0, \quad u(0)=u_{0}, \quad u=\left(1-\alpha \Delta_{x}\right) \bar{u},
\end{array}\right.
$$

on a $2 \mathrm{D}$ torus $\mathbb{T}^{2}=(0, L)^{2}$ with the forcing $g \in H^{1}\left(\mathbb{T}^{2}\right)$ and $\alpha, \gamma>0$. The system is studied in the phase space $H^{-1}\left(\mathbb{T}^{2}\right) \cap\{\operatorname{div} u=0\}=: H^{-1}$. We also assume that $\int_{\mathbb{T}^{2}}(u, \bar{u}, g) d x=0$. Here $\alpha=\alpha^{\prime} L^{2}$ and $\alpha^{\prime}>0$ is a small dimensionless parameter, so that $\bar{u}$ is a smoothed (filtered) vector field.

Alternatively, equations (1.4) can be considered as a particular case of the so-called Kelvin-Voight regularization of damped Navier-Stokes equations. Indeed, rewriting it in terms of the variable $\bar{u}$, we arrive at the equations

$$
\left\{\begin{array}{l}
\partial_{t} \bar{u}-\alpha \Delta_{x} \partial_{t} \bar{u}+\left(\bar{u}, \nabla_{x}\right) \bar{u}+\gamma \alpha \bar{u}=\gamma \alpha \Delta_{x} \bar{u}+g, \\
\operatorname{div} \bar{u}=0, \quad \bar{u}(0)=\bar{u}_{0},
\end{array}\right.
$$

which are damped Kelvin-Voight Navier-Stokes equations with the specific choice of Ekman damping parameter $\bar{\gamma}:=\gamma \alpha$ which coincides with the kinematic viscosity 
$v:=\gamma \alpha$, see $[4,21]$ for more details. In the present paper we restrict our attention to 2D space periodic case since only in this case the sharp lower bounds for the attractor's dimension can be obtained. The explicit upper bounds for this dimension in bounded and unbounded 3D domains will be given in the forthcoming paper [19].

Let us describe the results of this paper. In section 2 we prove that system (1.4) is dissipative in the phase space $H^{-1}\left(\mathbb{T}^{2}\right)$ and prove the existence of the global attractor. Since for $\alpha>0$ the convective term is a bounded (compact) perturbation, the existence of the global attractor is essentially an ODE result.

As far as the attractor is concerned, the choice of the phase space can vary, however, in the next section 3 the phase space $H^{-1}\left(\mathbb{T}^{2}\right)$ is most convenient for the estimates of the global Lyapunov exponents by means of the Constantin-Foias-Temam $N$-trace formula $[2,11,31]$.

The following two-sided order-sharp estimate (as $\alpha \rightarrow 0^{+}$) of the dimension of the global attractor $\mathscr{A}=\mathscr{A}_{\alpha}$ of system (1.4) is the main result of this work

$$
6.46 \cdot 10^{-7} \frac{\|\operatorname{curl} g\|_{L^{2}}^{2}}{\alpha \gamma^{4}} \leq \operatorname{dim}_{F} \mathscr{A} \leq \frac{1}{8 \pi} \frac{\|\operatorname{curl} g\|_{L^{2}}^{2}}{\alpha \gamma^{4}} .
$$

We observe that its looks somewhat similar to (1.3) with $v$ interchanged with $\alpha$.

While the right-hand side estimate is universal, the lower bound here holds as in (1.3) for a family of Kolmogorov right-hand sides $g=g_{s}$ specially chosen in section 4 . For this purpose we use the instability analysis for the family of generalized Kolmogorov flows generated by the family of right-hand sides

$$
g=g_{s}=\left\{\begin{array}{l}
f_{1}=\frac{1}{\sqrt{2} \pi} \gamma \lambda(s) \sin s x_{2} \\
g_{2}=0
\end{array}\right.
$$

where $s \in \mathbb{N}$. We show that the parameter $\lambda(s)$ can be chosen so that the unstable manifold of the stationary solution corresponding to $g_{s}$ has dimension $O\left(s^{2}\right)$, while for $s:=\alpha^{-1 / 2}$ we have

$$
\left\|\operatorname{curl} g_{s}\right\|_{L^{2}}=O(1) \quad \text { as } \alpha \rightarrow 0 .
$$

Since the unstable manifold lies in the global attractor, it follows that we have a lower bound for the dimension of the attractor $\mathscr{A}_{s}$ corresponding to $g=g_{s}$, which can be also written in an explicit form given on the left-hand side in (1.6).

Finally, in the Appendix in section 5 we prove the following spectral inequality that is an essential analytical ingredient in the proof of the upper bound for the dimension:

$$
\|\rho\|_{L^{2}} \leq \frac{1}{2 \sqrt{\pi}} m^{-1} n^{1 / 2}, \quad \rho(x)=\sum_{j=1}^{n}\left|u_{j}(x)\right|^{2},
$$

where

$$
u_{j}=\left(m^{2}-\Delta_{x}\right)^{-1 / 2} \psi_{j}
$$


and $\left\{\psi_{j}\right\}_{j=1}^{n}$ is an orthonormal family in $L^{2}\left(\mathbb{T}^{2}\right)$ and each $\psi_{j}$ has mean value zero. The inequality of this type is proved (among others) in [23] in the case of $\mathbb{R}^{d}$. The case of the torus requires a special treatment, since in this case the parameter $m^{2}$ can no longer be scaled out.

\section{Global attractor}

Theorem 2.1. There exist a unique solution $u=u(t) \in H^{-1}$ of system (1.4), which defines the semigroup of solution operators acting in $H^{-1}$ :

$$
S(t) u_{0}=u(t), \quad S(0) u_{0}=u_{0} .
$$

Proof. Since for $u \in H^{-1}$ we have $\bar{u} \in H^{1}$, it follows that the nonlinear term $\left(\bar{u}, \nabla_{x}\right) \bar{u}$ is bounded and Lipschitz in $H^{-1}$. Therefore the existence of a local in time unique solution is straightforward. Furthermore, the solution is continuous with respect to the initial data $u_{0} \in H^{-1}$. The fact that it exists for all $t>0$ follows from a priori estimates derived below.

Vorticity equation. Taking curl from both sides of (1.4) we arrive at the vorticity equation for $\omega=\operatorname{curl} u$

$$
\partial_{t} \omega+\left(\bar{u}, \nabla_{x}\right) \bar{\omega}+\gamma \omega=\operatorname{curl} g, \omega=\left(1-\alpha \Delta_{x}\right) \bar{\omega} .
$$

Multiplying this equation by $\bar{\omega}$ and integrating over $x$, we get

$$
\begin{gathered}
\frac{1}{2} \frac{d}{d t}\left(\|\bar{\omega}\|_{L^{2}}^{2}+\alpha\left\|\nabla_{x} \bar{\omega}\right\|_{L^{2}}^{2}\right)+\gamma\left(\|\bar{\omega}\|_{L^{2}}^{2}+\alpha\left\|\nabla_{x} \bar{\omega}\right\|_{L^{2}}^{2}\right)=(\operatorname{curl} g, \bar{\omega})= \\
=\left(g, \nabla_{x}^{\perp} \bar{\omega}\right) \leq\|g\|_{L^{2}}\left\|\nabla_{x} \bar{\omega}\right\|_{L^{2}} \leq \frac{1}{2 \alpha \gamma}\|g\|_{L^{2}}^{2}+\frac{\alpha \gamma}{2}\left\|\nabla_{x} \bar{\omega}\right\|_{L^{2}}^{2} .
\end{gathered}
$$

This gives by Gronwall's inequality the estimate

$$
\|\bar{\omega}(t)\|_{L^{2}}^{2}+\alpha\left\|\nabla_{x} \bar{\omega}(t)\right\|_{L^{2}}^{2} \leq \frac{1}{\alpha \gamma^{2}}\|g\|_{L^{2}}^{2},
$$

which holds for every trajectory $u(t)$ as $t \rightarrow \infty$.

Alternatively, without integration by parts of the curl on the right-hand side, we can write

$$
\|\operatorname{curl} g\|_{L^{2}}\|\bar{\omega}\|_{L^{2}} \leq \frac{1}{2 \gamma}\|\operatorname{curl} g\|_{L^{2}}^{2}+\frac{\gamma}{2}\|\bar{\omega}\|_{L^{2}}^{2},
$$

giving as $t \rightarrow \infty$

$$
\|\bar{\omega}(t)\|_{L^{2}}^{2}+\alpha\left\|\nabla_{x} \bar{\omega}(t)\right\|_{L^{2}}^{2} \leq \frac{1}{\gamma^{2}}\|\operatorname{curl} g\|_{L^{2}}^{2},
$$

so that as $t \rightarrow \infty$

$$
\|\bar{\omega}(t)\|_{L^{2}}^{2}+\alpha\left\|\nabla_{x} \bar{\omega}(t)\right\|_{L^{2}}^{2} \leq \frac{1}{\gamma^{2}} \min \left(\frac{\|g\|_{L^{2}}^{2}}{\alpha},\|\operatorname{curl} g\|_{L^{2}}^{2}\right)=: R_{0}^{2} .
$$


Proposition 2.2. For any $R_{1}>0$ and any $u_{0}$ with $\left\|u_{0}\right\|_{H^{-1}} \leq R_{1}$ there exists a time $T=T\left(R_{1}, R_{0}\right)$ such that for all $t \geq T\left(R_{1}, R_{0}\right)$ the solution $u(t)=S(t) u_{0}$ enters and never leaves the ball of radius $2 R_{0}$ in $H^{-1}$ :

$$
\|u(t)\|_{H^{-1}} \leq 2 R_{0} \quad \text { for } \quad t \geq T\left(R_{1}, R_{0}\right) .
$$

In other words, this ball is an absorbing set for $S(t)$ in $H^{-1}$.

Proof. The claim of the proposition follows from (2.2) (where we may want to drop the term with $\alpha$ on the left-hand side) and the following equivalence of the norms

$$
\|u\|_{H^{-1}} \sim\|\bar{u}\|_{H^{1}}=\left\|\nabla_{x} \bar{u}\right\|_{L^{2}}=\|\bar{\omega}\|_{L^{2}} .
$$

Asymptotic compactness and global attractor. To prove the asymptotic compactness of the semigroup $S(t)$ we construct an attracting compact set in the absorbing ball in $H^{-1}$. For this purpose we decompose $S(t)$ as follows

$$
S(t)=\Sigma(T)+S_{2}(t), \quad S_{2}(t)=S(t)-\Sigma(t),
$$

where $\Sigma(t)$ is exponentially contacting and $S_{2}(t)$ is uniformly compact. This decomposition (and its more elaborate variants) is very useful for dissipative hyperbolic problems $[2,7,31]$ and the original idea goes back to [14].

Let $\Sigma(t)$ be the solution (semi)group of the linear equation

$$
\partial_{t} v+\gamma v=0, \quad \operatorname{div} v=0, \quad v(0)=u_{0},
$$

and $w$ (playing the role of $\left.S_{2}(t)\right)$ is the solution of

$$
\partial_{t} w+\gamma w+\nabla_{x} p=G(t):=-\left(\bar{u}, \nabla_{x}\right) \bar{u}+g, \quad \operatorname{div} w=0, \quad w(0)=0,
$$

where, of course, $u=v+w$. Obviously, $v(t)=e^{-\gamma t} u_{0}$ is exponentially decaying in $H^{-1}$ (and in every $H^{s}$ ). The right-hand side $G(t)$ in the "linear" equation for $w$ is bounded in $H^{-\delta}$ for every $\delta>0$ uniformly in $t$. In fact, since $\bar{u}(t)$ is bounded in $H^{1}$, it follows from the Sobolev imbedding theorem and Hölder's inequality that $\left(\bar{u}, \nabla_{x}\right) \bar{u}$ is bounded in $L^{2-\varepsilon}$ for $\varepsilon>0$, while, in turn, by duality $L^{2-\varepsilon} \subset H^{-\delta}, \delta=\delta(\varepsilon)=\varepsilon /(2-\varepsilon)$.

Taking into account that $w(0)=0$ we see that the solution $w$ is bounded in $H^{-\delta}$ uniformly with respect to $t$, and since the imbedding $H^{-\delta}\left(\mathbb{T}^{2}\right) \subset H^{-1}\left(\mathbb{T}^{2}\right)$ is compact, the asymptotic compactness of the solution semigroup $S(t)$ is established.

We recall the definition of the global attractor.

Definition 2.3. Let $S(t), t \geq 0$, be a semigroup in a Banach space $H$. The set $\mathscr{A} \subset H$ is a global attractor of the semigroup $S(t)$ if

1) The set $\mathscr{A}$ is compact in $H$.

2) It is strictly invariant: $S(t) \mathscr{A}=\mathscr{A}$.

3) It attracts the images of bounded sets in $H$ as $t \rightarrow \infty$, i.e., for every bounded set $B \subset H$ and every neighborhood $O(\mathscr{A})$ of the set $\mathscr{A}$ in $H$ there exists $T=T(B, O)$ such that for all $t \geq T$

$$
S(t) B \subset O(\mathscr{A}) .
$$


The following general result (see, for instance, $[2,7,31]$ ) gives sufficient conditions for the existence of a global attractor.

Theorem 2.4. Let $S(t)$ be a semigroup in a Banach space H. Suppose that

1) $S(t)$ possesses a bounded absorbing ball $B \subset H$;

2) For every fixed $t \geq 0$ the map $S(t): B \rightarrow H$ is continuous.

3) $S(t)$ is asymptotically compact.

Then the semigroup $S(t)$ possesses a global attractor $\mathscr{A} \subset B$. Moreover, the attractor $\mathscr{A}$ has the following structure:

$$
\mathscr{A}=\left.\mathscr{K}\right|_{t=0},
$$

where $\mathscr{K} \subset L^{\infty}(\mathbb{R}, H)$ is the set of complete trajectories $u: \mathbb{R} \rightarrow H$ of semigroup $S(t)$ which are defined for all $t \in \mathbb{R}$ and bounded.

We are now prepared to state the main result of this section, whose proof directly follows from Theorem 2.4 since its assumptions were verified above.

Theorem 2.5. The semigroup $S(t)$ corresponding to (1.4) possesses in the phase space $H^{-1}$ a global attractor $\mathscr{A}$.

\section{Dimension estimate}

Theorem 3.1. The global attractor $\mathscr{A}$ corresponding to the regularized damped Euler system (1.4) has finite fractal dimension

$$
\operatorname{dim}_{F} \mathscr{A} \leq \frac{1}{8 \pi} \frac{\|\operatorname{curl} g\|_{L^{2}}^{2}}{\alpha \gamma^{4}} .
$$

Proof. Note that the differentiability of the solution semigroup $S(t): H^{-1} \rightarrow H^{-1}$ is obvious here, so we only need to estimate the traces. The equation of variations for equation (1.4) reads:

$$
\partial_{t} \theta=-\gamma \theta-\left(\bar{u}, \nabla_{x}\right) \bar{\theta}-\left(\bar{\theta}, \nabla_{x}\right) \bar{u}-\nabla_{x} p=: L_{u(t)} \theta, \quad \operatorname{div} \theta=0 .
$$

We will estimate volume contraction factor in the space $H^{-1}$ endowed with the norm

$$
\|\theta\|_{\alpha}^{2}:=\|\bar{\theta}\|_{L^{2}}^{2}+\alpha\left\|\nabla_{x} \bar{\theta}\right\|_{L^{2}}^{2}=\|\bar{\theta}\|_{L^{2}}^{2}+\alpha\|\operatorname{curl} \bar{\theta}\|_{L^{2}}^{2}=\left\|\left(1-\alpha \Delta_{x}\right)^{-1 / 2} \theta\right\|^{2}
$$

and scalar product

$$
(\theta, \xi)_{\alpha}=\left(\left(1-\alpha \Delta_{x}\right)^{-1 / 2} \theta,\left(1-\alpha \Delta_{x}\right)^{-1 / 2} \xi\right) .
$$

The numbers $q(n)$ (the sums of the first $n$ global Lyapunov exponents) are estimated/defined as follows [31]

$$
q(n) \leq(:=) \limsup _{t \rightarrow \infty} \sup _{u(t) \in \mathscr{A}} \sup _{\left\{\theta_{j}\right\}_{j=1}^{n}} \frac{1}{t} \int_{0}^{t} \sum_{j=1}^{n}\left(L_{u(\tau)} \theta_{j}, \theta_{j}\right)_{\alpha} d \tau,
$$


where $\left\{\theta_{j}\right\}_{j=1}^{n}$ is an orthonormal family with respect to $(\cdot, \cdot)_{\alpha}$ :

$$
\left(\theta_{i}, \theta_{j}\right)_{\alpha}=\delta_{i j}, \quad \operatorname{div} \theta_{j}=0
$$

and $u(t)$ is an arbitrary trajectory on the attractor.

Then,

$$
\begin{array}{r}
\sum_{j=1}^{n}\left(L_{u(t)} \theta_{j}, \theta_{j}\right)_{\alpha}=\sum_{j=1}^{n}\left(L_{u(t)} \theta_{j}, \bar{\theta}_{j}\right)= \\
=-\sum_{j=1}^{n} \gamma\left\|\theta_{j}\right\|_{\alpha}^{2}-\sum_{j=1}^{n}\left(\left(\bar{\theta}_{j}, \nabla_{x}\right) \bar{u}, \bar{\theta}_{j}\right)= \\
=-\gamma n-\sum_{j=1}^{n}\left(\left(\bar{\theta}_{j}, \nabla_{x}\right) \bar{u}, \bar{\theta}_{j}\right) .
\end{array}
$$

Next, $\theta_{j} \in H^{-1}$ are such that the family $\left\{\left(1-\alpha \Delta_{x}\right)^{-1 / 2} \theta_{j}\right\}_{j=1}^{n}=:\left\{\psi_{j}\right\}_{j=1}^{n}$ is orthonormal in $L^{2}$. By definition

$$
\bar{\theta}_{j}=\left(1-\alpha \Delta_{x}\right)^{-1} \theta_{j}=\left(1-\alpha \Delta_{x}\right)^{-1 / 2} \psi_{j},
$$

and therefore for the function

$$
\rho(x)=\sum_{j=1}^{n}\left|\bar{\theta}_{j}(x)\right|^{2}
$$

in view of (5.2) (with $p=2$ ) and (5.3) we have the estimate

$$
\|\rho\|_{L^{2}} \leq \mathrm{B}_{2} \frac{n^{1 / 2}}{\sqrt{\alpha}}, \quad \mathrm{B}_{2} \leq \frac{1}{2 \sqrt{\pi}} .
$$

Since $\operatorname{div} \bar{u}=0$, we have pointwise

$$
\left|\left(\bar{\theta}, \nabla_{x}\right) \bar{u} \cdot \bar{\theta}\right| \leq \frac{1}{\sqrt{2}}|\bar{\theta}(x)|^{2}\left|\nabla_{x} \bar{u}(x)\right|
$$

and using (3.3) and (2.2) we obtain

$$
\begin{array}{r}
\sum_{j=1}^{n}\left|\left(\left(\bar{\theta}_{j}, \nabla_{x}\right) \bar{u}, \bar{\theta}_{j}\right)\right| \leq \frac{1}{\sqrt{2}} \int_{\mathbb{T}^{2}} \rho(x)\left|\nabla_{x} \bar{u}\right| d x \leq \\
\leq \frac{1}{\sqrt{2}}\|\rho\|_{L^{2}}\left\|\nabla_{x} \bar{u}\right\|_{L^{2}}=\frac{1}{\sqrt{2}}\|\rho\|_{L^{2}}\|\bar{\omega}\|_{L^{2}} \leq \\
\leq \frac{\mathrm{B}_{2}}{\sqrt{2}} \frac{n^{1 / 2}}{\sqrt{\alpha}} \frac{\|\operatorname{curl} g\|_{L^{2}}}{\gamma} .
\end{array}
$$


Since all our estimates are uniform with respect time and the $\alpha$-orthonormal family (3.2), it follows that

$$
q(n) \leq-\gamma n+\frac{\mathrm{B}_{2}}{\sqrt{2}} \frac{n^{1 / 2}}{\sqrt{\alpha}} \frac{\|\operatorname{curl} g\|_{L^{2}}}{\gamma} .
$$

The number $n^{*}$ such that for which $q\left(n^{*}\right)=0$ and $q(n)<0$ for $n>n^{*}$ is the upper bound both for the Hausdorff $[2,31]$ and the fractal $[5,6]$ dimension of the global attractor $\mathscr{A}$ :

$$
\operatorname{dim}_{F} \mathscr{A} \leq \frac{\mathrm{B}_{2}^{2}}{2} \frac{\|\operatorname{curl} g\|_{L^{2}}^{2}}{\alpha \gamma^{4}} \leq \frac{1}{8 \pi} \frac{\|\operatorname{curl} g\|_{L^{2}}^{2}}{\alpha \gamma^{4}}
$$

\section{A sharp lower bound}

In this section we derive a sharp lower bound for the dimension of the global attractor $\mathscr{A}$ based on the generalized Kolmogorov flows [26, 25, 34].

We consider the vorticity equation (2.1)

$$
\partial_{t} \omega+J\left(\left(\Delta_{x}-\alpha \Delta_{x}^{2}\right)^{-1} \omega,\left(1-\alpha \Delta_{x}\right)^{-1} \omega\right)+\gamma \omega=\operatorname{curl} g,
$$

where $J$ is the Jacobian operator

$$
J(a, b)=\nabla^{\perp} a \cdot \nabla b=\partial_{x_{1}} a \partial_{x_{2}} b-\partial_{x_{2}} a \partial_{x_{1}} b .
$$

Next, let $g=g_{s}$ be a family of right-hand sides

$$
g=g_{s}=\left\{\begin{array}{l}
f_{1}=\frac{1}{\sqrt{2} \pi} \gamma \lambda(s) \sin s x_{2}, \\
g_{2}=0,
\end{array}\right.
$$

where $s \in \mathbb{N}$, and $\lambda(s)$ is a parameter to be chosen later. Then

$$
\operatorname{curl} g_{s}=-\frac{1}{\sqrt{2} \pi} \gamma \lambda(s) s \cos s x_{2},
$$

and

$$
\left\|g_{s}\right\|_{L^{2}}^{2}=\gamma^{2} \lambda(s)^{2}, \quad\left\|\operatorname{curl} g_{s}\right\|_{L^{2}}^{2}=\gamma^{2} \lambda(s)^{2} s^{2} .
$$

Corresponding to the family (4.3) is the family of stationary solutions

$$
\omega_{s}=-\frac{1}{\sqrt{2} \pi} \lambda(s) s \cos s x_{2}
$$

of equation (4.1). Since $\omega_{s}$ depends only on $x_{2}$, the nonlinear term vanishes:

$$
J\left(\left(\Delta_{x}-\alpha \Delta_{x}^{2}\right)^{-1} \omega_{s},\left(1-\alpha \Delta_{x}\right)^{-1} \omega_{s}\right) \equiv 0 .
$$


We linearize (4.1) about the stationary solution (4.5) and consider the eigenvalue problem

$$
\begin{aligned}
\mathcal{L}_{S} \omega & :=J\left(\left(\Delta_{x}-\alpha \Delta_{x}^{2}\right)^{-1} \omega_{s},\left(1-\alpha \Delta_{x}\right)^{-1} \omega\right)+ \\
& +J\left(\left(\Delta_{x}-\alpha \Delta_{x}^{2}\right)^{-1} \omega,\left(1-\alpha \Delta_{x}\right)^{-1} \omega_{s}\right)+\gamma \omega=-\sigma \omega .
\end{aligned}
$$

The solutions with $\operatorname{Re} \sigma>0$ will be unstable eigenmodes.

We use the orthonormal basis of trigonometric functions, which are the eigenfunctions of the Laplacian,

$$
\begin{array}{r}
\left\{\frac{1}{\sqrt{2} \pi} \sin k x, \frac{1}{\sqrt{2} \pi} \cos k x\right\}, \quad k x=k_{1} x_{1}+k_{2} x_{2}, \\
k \in \mathbb{Z}_{+}^{2}=\left\{k \in \mathbb{Z}_{0}^{2}, k_{1} \geq 0, k_{2} \geq 0\right\} \cup\left\{k \in \mathbb{Z}_{0}^{2}, k_{1} \geq 1, k_{2} \leq 0\right\}
\end{array}
$$

and write

$$
\omega(x)=\frac{1}{\sqrt{2} \pi} \sum_{k \in \mathbb{Z}_{+}^{2}} a_{k} \cos k x+b_{k} \sin k x .
$$

Substituting this into (4.6) and using the equality $J(a, b)=-J(b, a)$ we obtain

$$
\begin{gathered}
\frac{s \lambda(s)}{\sqrt{2} \pi\left(s^{2}+\alpha s^{4}\right)} \sum_{k \in \mathbb{Z}_{+}^{2}}\left(\frac{k^{2}-s^{2}}{k^{2}+\alpha k^{4}}\right) J\left(\cos s x_{2}, a_{k} \cos k x+b_{k} \sin k x\right)+ \\
+(\gamma+\sigma) \sum_{k \in \mathbb{Z}_{+}^{2}}\left(a_{k} \cos k x+b_{k} \sin k x\right)=0 .
\end{gathered}
$$

Next, we have the following two similar formulas

$$
\begin{array}{r}
J\left(\cos s x_{2}, \cos \left(k_{1} x_{1}+k_{2} x_{2}\right)\right)=-k_{1} s \sin s x_{2} \sin \left(k_{1} x_{1}+k_{2} x_{2}\right)= \\
=\frac{k_{1} s}{2}\left(\cos \left(k_{1} x_{1}+\left(k_{2}+s\right) x_{2}\right)-\cos \left(k_{1} x_{1}+\left(k_{2}-s\right) x_{2}\right),\right. \\
J\left(\cos s x_{2}, \sin \left(k_{1} x_{1}+k_{2} x_{2}\right)\right)=k_{1} s \sin s x_{2} \cos \left(k_{1} x_{1}+k_{2} x_{2}\right)= \\
=\frac{k_{1} s}{2}\left(\sin \left(k_{1} x_{1}+\left(k_{2}+s\right) x_{2}\right)-\sin \left(k_{1} x_{1}+\left(k_{2}-s\right) x_{2}\right),\right.
\end{array}
$$

which we substitute into (4.7) and collect the terms with $\cos \left(k_{1} x_{1}+k_{2} x_{2}\right)$. We obtain the following equation for the coefficients $a_{k_{1}, k_{2}}$ (the equation for $b_{k_{1}, k_{2}}$ is exactly the same):

$$
\begin{aligned}
& -\Lambda(s) k_{1}\left(\frac{k_{1}^{2}+\left(k_{2}+s\right)^{2}-s^{2}}{\left.k_{1}^{2}+\left(k_{2}+s\right)^{2}+\alpha\left(k_{1}^{2}+\left(k_{2}+s\right)^{2}\right)^{2}\right)}\right) a_{k_{1} k_{2}+s}+ \\
& +\Lambda(s) k_{1}\left(\frac{k_{1}^{2}+\left(k_{2}-s\right)^{2}-s^{2}}{\left.k_{1}^{2}+\left(k_{2}-s\right)^{2}+\alpha\left(k_{1}^{2}+\left(k_{2}-s\right)^{2}\right)^{2}\right)}\right) a_{k_{1} k_{2}-s}+(\gamma+\sigma) a_{k_{1} k_{2}}=0,
\end{aligned}
$$

where

$$
\Lambda=\Lambda(s):=\frac{s^{2} \lambda(s)}{2 \sqrt{2} \pi\left(s^{2}+\alpha s^{4}\right)}=\frac{\lambda(s)}{2 \sqrt{2} \pi\left(1+\alpha s^{2}\right)}
$$


We set here

$$
a_{k_{1} k_{2}}\left(\frac{k^{2}-s^{2}}{k^{2}+\alpha k^{4}}\right)=: c_{k_{1} k_{2}},
$$

and setting further

$$
\begin{gathered}
k_{1}=t, \quad k_{2}=s n+r, \quad \text { and } \quad c_{t s n+r}=e_{n}, \\
t=1,2, \ldots, \quad r \in \mathbb{Z}, \quad r_{\min }<r<r_{\max },
\end{gathered}
$$

where the numbers $r_{\min }, r_{\max }$ satisfy $r_{\max }-r_{\min }<s$ and will be specified below we obtain for each $t$ and $r$ the following three-term recurrence relation:

$$
d_{n} e_{n}+e_{n-1}-e_{n+1}=0, \quad n=0, \pm 1, \pm 2, \ldots,
$$

where

$$
d_{n}=\frac{\left(t^{2}+(s n+r)^{2}+\alpha\left(t^{2}+(s n+r)^{2}\right)^{2}\right)(\gamma+\sigma)}{\Lambda t\left(t^{2}+(s n+r)^{2}-s^{2}\right)} .
$$

We look for non-trivial decaying solutions $\left\{e_{n}\right\}$ of (4.9), (4.10). Each non-trivial decaying solution with $\operatorname{Re} \sigma>0$ produces an unstable eigenfunction $\omega$ of the eigenvalue problem (4.6).

Theorem 4.1. Given an integer $s>0$ let a fixed pair of integers $t, r$ belong to a bounded region $A(\delta)$ defined by conditions

$$
\begin{array}{r}
t^{2}+r^{2}<s^{2} / 3, \quad t^{2}+(-s+r)^{2}>s^{2}, \quad t^{2}+(s+r)^{2}>s^{2}, \quad t \geq \delta s, \\
r_{\min }<r<r_{\max }, \quad r_{\min }=-\frac{s}{6}, r_{\max }=\frac{s}{6}, \quad 0<\delta<1 / \sqrt{3} .
\end{array}
$$

For any $\Lambda>0$ there exists a unique real eigenvalue $\sigma=\sigma(\Lambda)$, which increases monotonically as $\Lambda \rightarrow \infty$ and satisfies the inequality

$$
\Lambda \frac{21 \sqrt{2} \delta^{2} s}{55\left(1+\alpha s^{2}\right)}-\gamma \leq \sigma(\Lambda) \leq \Lambda \frac{\sqrt{2} \delta^{-1} s}{\left(1+\alpha s^{2}\right)}-\gamma .
$$

The unique $\Lambda_{0}=\Lambda_{0}(s)$ solving the equation

$$
\sigma\left(\Lambda_{0}\right)=0
$$

satisfies the two-sided estimate

$$
\frac{\gamma \delta}{\sqrt{2}} \frac{\left(1+\alpha s^{2}\right)}{s}<\Lambda_{0}<\frac{55 \gamma \delta^{-2}}{21 \sqrt{2}} \frac{1+\alpha s^{2}}{s} .
$$

Proof. We shall follow quite closely the argument in [17] and first observe that the following inequalities hold for any $(t, r)$ satisfying (4.11):

$$
\begin{aligned}
& s^{2} \leq t^{2}+(-s+r)^{2}=\operatorname{dist}((0, s),(t, r))^{2} \leq \operatorname{dist}((0, s), C)^{2}=(5 / 3) s^{2}, \\
& s^{2} \leq t^{2}+(s+r)^{2}=\operatorname{dist}((0,-s),(t, r))^{2} \leq \operatorname{dist}((0,-s), B)^{2}=(5 / 3) s^{2},
\end{aligned}
$$


where $B=(\sqrt{11} s / 6, s / 6)$ and $C=(\sqrt{11} s / 6,-s / 6)$.

In view of (4.11) for any real $\sigma$ satisfying $\sigma>-\gamma$ we have in (4.9), (4.10)

$$
d_{0}<0, d_{n}>0 \text { for } n \neq 0 \text { and } \lim _{|n| \rightarrow \infty} d_{n}=\infty \text {. }
$$

The main tool in the analysis of (4.9) are continued fractions and a variant of Pincherle's theorem (see [20], [25], [26], [34]) saying that under condition (4.15) recurrence relation (4.9) has a decaying solution $\left\{e_{n}\right\}$ with $\lim _{|n| \rightarrow \infty} e_{n}=0$ if and only if

$$
-d_{0}=\frac{1}{d_{-1}+\frac{1}{d_{-2}+\ldots}}+\frac{1}{d_{1}+\frac{1}{d_{2}+\ldots}} .
$$

Next, we set

$$
\begin{gathered}
f(\sigma)=-d_{0}=\frac{(\gamma+\sigma)\left(t^{2}+r^{2}+\alpha\left(t^{2}+r^{2}\right)^{2}\right)}{\Lambda t\left(s^{2}-\left(t^{2}+r^{2}\right)\right)}, \\
g(\sigma)=\frac{1}{d_{-1}+\frac{1}{d_{-2}+\ldots}}+\frac{1}{d_{1}+\frac{1}{d_{2}+\ldots}} .
\end{gathered}
$$

It follows from (4.17) that

$$
f(-\gamma)=0, \quad \text { and } \quad f(\sigma) \rightarrow \infty \quad \text { as } \quad \sigma \rightarrow \infty,
$$

while (4.18) and (4.10) give that

$$
g(\sigma)<\frac{1}{d_{-1}}+\frac{1}{d_{1}}, \quad g(\sigma) \rightarrow 0 \quad \text { as } \quad \sigma \rightarrow \infty .
$$

Hence, there exists a $\sigma>-\gamma$ such that

$$
f(\sigma)=g(\sigma)
$$

From elementary properties of continued fractions we deduce as in [25], [34] that $\sigma=\sigma(\Lambda)$ so obtained is unique and increases monotonically with $\Lambda$.

To establish (4.12) we deduce from (4.19) and (4.18) that

$$
\frac{1}{d_{-1}+\frac{1}{d_{-2}}}+\frac{1}{d_{1}+\frac{1}{d_{2}}}<f(\sigma)<\frac{1}{d_{-1}}+\frac{1}{d_{1}} .
$$

Using (4.11) we see from (4.10) that

$$
\begin{aligned}
& \frac{1}{d_{ \pm 1}}=\frac{\Lambda t}{\gamma+\sigma} \cdot \frac{t^{2}+(s \pm r)^{2}-s^{2}}{\left.t^{2}+(s \pm r)^{2}+\alpha\left(t^{2}+(s \pm r)^{2}\right)^{2}\right)} \leq \\
& \leq \frac{\Lambda t}{\gamma+\sigma} \cdot \frac{1}{1+\alpha\left(t^{2}+(s \pm r)^{2}\right)} \leq \frac{\Lambda t}{\gamma+\sigma} \cdot \frac{1}{1+\alpha s^{2}}
\end{aligned}
$$


and therefore from the right-hand side inequality in (4.20) it follows that

$$
f(\sigma)=\frac{(\gamma+\sigma)\left(t^{2}+r^{2}+\alpha\left(t^{2}+r^{2}\right)^{2}\right)}{\Lambda t\left(s^{2}-\left(t^{2}+r^{2}\right)\right)}<\frac{1}{d_{-1}}+\frac{1}{d_{1}}<\frac{2 \Lambda t}{\gamma+\sigma} \cdot \frac{1}{1+\alpha s^{2}},
$$

which gives the right-hand side inequality in (4.12):

$$
\left(1+\alpha s^{2}\right)(\gamma+\sigma)^{2} \leq \frac{2 \Lambda^{2} t^{2}\left(s^{2}-\left(t^{2}+r^{2}\right)\right)}{t^{2}+r^{2}+\alpha\left(t^{2}+r^{2}\right)^{2}} \leq \frac{2 \Lambda^{2} t^{2} s^{2}}{t^{2}+\alpha t^{4}}=\frac{2 \Lambda^{2} s^{2}}{1+\alpha t^{2}} \leq \frac{2 \Lambda^{2} \delta^{-2} s^{2}}{1+\alpha s^{2}} .
$$

From the left-hand side inequality in (4.20), where $d_{-1}, d_{1}, d_{-2}, d_{2}, f>0$, we see that

$$
f d_{1}+\frac{f}{d_{2}}>1 \text { and } f d_{-1}+\frac{f}{d_{-2}}>1 .
$$

Next, it follows from (4.11) that $4 s r \leq 2 s^{2} / 3$ and $|2 s+r| \geq 11 s / 6$. Therefore

$$
\begin{aligned}
& \quad \frac{f}{d_{2}}=\frac{\left(t^{2}+r^{2}+\alpha\left(t^{2}+r^{2}\right)^{2}\right)\left(t^{2}+(2 s+r)^{2}-s^{2}\right)}{\left(s^{2}-\left(t^{2}+r^{2}\right)\right)\left(t^{2}+(2 s+r)^{2}+\alpha\left(t^{2}+(2 s+r)^{2}\right)^{2}\right)}< \\
& <\frac{\left(s^{2} / 3+\alpha s^{4} / 9\right) 4 s^{2}}{2 s^{2} / 3\left((11 / 6)^{2} s^{2}+\alpha(11 / 6)^{4} s^{4}\right)}=\frac{2(1+t / 3)}{(11 / 6)^{2}+t(11 / 6)^{4}}{ }_{t=\alpha s^{2}} \leq \frac{72}{121} .
\end{aligned}
$$

Along with (4.21) this implies that $f d_{1}>49 / 121$, which for $r \geq 0$ gives that

$$
\begin{aligned}
& \frac{49}{121}<f d_{1}= \\
= & \frac{(\gamma+\sigma)^{2}}{\Lambda^{2}} \frac{\left(t^{2}+r^{2}+\alpha\left(t^{2}+r^{2}\right)^{2}\right)\left(t^{2}+(s+r)^{2}+\alpha\left(t^{2}+(s+r)^{2}\right)^{2}\right)}{t^{2}\left(s^{2}-\left(t^{2}+r^{2}\right)\right)\left(t^{2}+(s+r)^{2}-s^{2}\right)}< \\
< & \frac{(\gamma+\sigma)^{2}}{\Lambda^{2}} \frac{\left(s^{2} / 3+\alpha s^{4} / 9\right)\left(5 s^{2} / 3+\alpha s^{4} 25 / 9\right)}{t^{2}(2 / 3) s^{2} t^{2}}< \\
< & \frac{25}{18} \frac{(\gamma+\sigma)^{2}}{\Lambda^{2}} \frac{\left(1+\alpha s^{2}\right)^{2}}{\delta^{4} s^{2}},
\end{aligned}
$$

and proves the left-hand side inequality in (4.12). For $r<0$ we use $d_{-1}$ instead of $d_{1}$.

Finally, estimate (4.13) follows from (4.12) with $\sigma=0$.

This result has the following important implications for the attractors of the damped doubly regularized Euler equations (1.4). Namely, it says that estimate (3.1) is ordersharp in the limit as $\alpha \rightarrow 0^{+}$.

Corollary 4.2. The parameter $\lambda(s)$ in the family (4.2) can be chosen so that the dimension of the corresponding global attractor $\mathscr{A}=\mathscr{A}_{s}$ satisfies

$$
\operatorname{dim} \mathscr{A} \geq \mathrm{c}_{1} \frac{\|\operatorname{curl} g\|_{L^{2}}^{2}}{\alpha \gamma^{4}} \quad \mathrm{c}_{1}>6.46 \cdot 10^{-7} .
$$


Proof. Writing (4.13) in terms of $\lambda(s)$ (see (4.8)) we see that for

$$
\lambda(s)=\frac{110 \pi}{21} \gamma \delta^{-2} \frac{\left(1+\alpha s^{2}\right)^{2}}{s}
$$

each point in $(t, r)$-plane satisfying (4.11) produces an unstable (positive) eigenvalue $\sigma$ of multiplicity two (the equation for the coefficients $b_{k}$ is the same). Denoting by $d(s)$ the number of points of the integer lattice inside the region $A(\delta)$ we obviously have

$$
d(s):=\#\left\{(t, r) \in D(s)=\mathbb{Z}^{2} \cap A(\delta)\right\} \simeq a(\delta) \cdot s^{2} \quad \text { as } \quad s \rightarrow \infty,
$$

where $a(\delta) \cdot s^{2}=|A(\delta)|$ is the area of the region $A(\delta)$ (see Fig.1 in [17]). Therefore the dimension of the unstable manifold around the stationary solution $\omega_{s}$ in (4.5) is at least $2 a(\delta) \cdot s^{2}$. The solutions lying on this unstable manifold are bounded on the entire time axis $t \in \mathbb{R}$, since they tend to $\omega_{s}$ as $t \rightarrow-\infty$ and all solutions are bounded as $t \rightarrow \infty$ in view of (2.2). Therefore representation (2.3) implies that [2]

$$
\operatorname{dim} A \geq 2 d(s) \simeq 2 a(\delta) \cdot s^{2} .
$$

It remains to express this lower bound in terms of the physical parameters of the system. So far $s$ was an arbitrary (large) parameter. We now set

$$
s:=\frac{1}{\sqrt{\alpha}}
$$

and see from (4.4) that

$$
\left\|\operatorname{curl} g_{s}\right\|_{L^{2}}^{2}=\gamma^{2} \lambda(s)^{2} s^{2}=\left(\frac{110 \pi}{21}\right)^{2} \gamma^{4} \delta^{-4}\left(1+\alpha s^{2}\right)^{4}=\left(\frac{110 \pi}{21}\right)^{2} \gamma^{4} \frac{2^{4}}{\delta^{4}},
$$

and

$$
\frac{\left\|\operatorname{curl} g_{s}\right\|_{L^{2}}^{2}}{\alpha \gamma^{4}}=\frac{1}{\alpha}\left(\frac{110 \pi}{21}\right)^{2} \frac{2^{4}}{\delta^{4}} .
$$

We finally obtain that (4.22) can be written in the form

$$
\operatorname{dim} \mathscr{A} \geq \max _{0<\delta<1 / \sqrt{3}} a(\delta) \delta^{4} \frac{1}{8}\left(\frac{21}{110 \pi}\right)^{2} \frac{\left\|\operatorname{curl} g_{s}\right\|_{L^{2}}^{2}}{\alpha \gamma^{4}}=6.46 \cdot 10^{-7} \frac{\left\|\operatorname{curl} g_{s}\right\|_{L^{2}}^{2}}{\alpha \gamma^{4}},
$$

where we optimized the result with respect to $\delta \in\left(0,3^{-1 / 2}\right)$

\section{Appendix}

We first recall a result in [23], which we formulate for the torus $\mathbb{T}^{2}$. 
Theorem 5.1. Let a family $\left\{\psi_{i}\right\}_{i=1}^{n}$ be orthonormal in $L^{2}\left(\mathbb{T}^{2}\right)$ and let $\int_{\mathbb{T}^{2}} \psi_{i}(x) d x=0$. Then the function

$$
\rho(x)=\sum_{i=1}^{n}\left|u_{i}(x)\right|^{2},
$$

where

$$
u_{i}=\left(m^{2}-\Delta_{x}\right)^{-1 / 2} \psi_{i},
$$

satisfies for $1 \leq p<\infty$ the estimate

$$
\|\rho\|_{L^{p}} \leq \mathrm{B}_{p} m^{-2 / p} n^{1 / p} .
$$

In our notation this estimate reads as follows.

Corollary 5.2. Let a family $\left\{\psi_{i}\right\}_{i=1}^{n}$ be orthonormal in $L^{2}\left(\mathbb{T}^{2}\right)$. Then the function

$$
\rho(x)=\sum_{i=1}^{n}\left|\bar{\theta}_{i}(x)\right|^{2},
$$

where

$$
\bar{\theta}_{i}=\left(1-\alpha \Delta_{x}\right)^{-1 / 2} \psi_{i}
$$

satisfies for $1 \leq p<\infty$ the estimate

$$
\|\rho\|_{L^{p}} \leq \mathrm{B}_{p} \alpha^{1 / p-1} n^{1 / p} .
$$

Proof. We write

$$
\bar{\theta}_{i}=\left(1-\alpha \Delta_{x}\right)^{-1 / 2} \psi_{i}=\left(\frac{1}{\alpha}-\Delta_{x}\right)^{-1 / 2} \frac{\psi_{i}}{\sqrt{\alpha}},
$$

and (5.1) gives (5.2):

$$
\left\|\alpha \sum_{i=1}^{n}\left|\bar{\theta}_{i}\right|^{2}\right\|_{L^{p}} \leq \mathrm{B}_{p} \alpha^{1 / p} n^{1 / p} .
$$

Next, we prove Theorem 5.1 for $p=2$ and give the estimate of the constant.

Proposition 5.3. Under the assumptions of Theorem 5.1 it holds for $p=2$

$$
\|\rho\|_{L^{2}} \leq \mathrm{B}_{2} m^{-1} n^{1 / 2}, \quad \mathrm{~B}_{2} \leq \frac{1}{2 \sqrt{\pi}} .
$$

Proof. We follow the idea in [23]. The main technical difference being that in the discrete case we cannot scale out $m$ and therefore we have to estimate the corresponding series over $\mathbb{Z}_{0}^{2}$ for all $m>0$. This can be done for all $1 \leq p<\infty$, but each case requires a special treatment (at least in our proof). 
For a non-negative function $V=V(x) \in L^{\infty}$ we set

$$
H=V^{1 / 2}\left(m^{2}-\Delta_{x}\right)^{-1 / 2}, \quad H^{*}=\left(m^{2}-\Delta_{x}\right)^{-1 / 2} V^{1 / 2} .
$$

We further set $K=H^{*} H$ and claim that

$$
\operatorname{Tr} K^{2} \leq \frac{1}{4 \pi} \frac{1}{m^{2}}\|V\|_{L^{2}}^{2} .
$$

In fact,

$$
\begin{aligned}
\operatorname{Tr} K^{2}= & \operatorname{Tr}\left(\left(m^{2}-\Delta_{x}\right)^{-1 / 2} V\left(m^{2}-\Delta_{x}\right)^{-1 / 2}\right)^{2} \leq \\
& \leq \operatorname{Tr}\left(\left(m^{2}-\Delta_{x}\right)^{-1} V^{2}\left(m^{2}-\Delta_{x}\right)^{-1}\right)= \\
& =\operatorname{Tr}\left(V^{2}\left(m^{2}-\Delta_{x}\right)^{-2}\right)
\end{aligned}
$$

where we used the Araki-Lieb-Thirring inequality for traces $[1,13]$ :

$$
\operatorname{Tr}\left(B A^{2} B\right)^{p} \leq \operatorname{Tr}\left(B^{p} A^{2 p} B^{p}\right),
$$

and the cyclicity property of the trace. Using the basis of orthonormal eigenfunctions of the Laplacian $(2 \pi)^{-1} e^{i k x}, k \in \mathbb{Z}_{0}^{2}=\mathbb{Z}^{2} \backslash\{0\}$ in view of (5.6) below we find that

$$
\begin{aligned}
\operatorname{Tr} K^{2} & \leq \operatorname{Tr}\left(V^{2}\left(m^{2}-\Delta_{x}\right)^{-2}\right)= \\
& =\frac{1}{4 \pi^{2}} \sum_{k \in \mathbb{Z}_{0}^{2}} \frac{1}{\left(|k|^{2}+m^{2}\right)^{2}} \int_{\mathbb{T}^{2}} V^{2}(x) d x \leq \frac{1}{4 \pi} \frac{1}{m^{2}}\|V\|_{L^{2}}^{2} .
\end{aligned}
$$

We can now complete the proof as in [23]. We observe that

$$
\int_{\mathbb{T}^{2}} \rho(x) V(x) d x=\sum_{i=1}^{n}\left\|H \psi_{i}\right\|_{L^{2}}^{2},
$$

and in view of orthonormality and the variational principle

$$
\sum_{i=1}^{n}\left\|H \psi_{i}\right\|_{L^{2}}^{2} \leq \sum_{i=1}^{n} \lambda_{i}
$$

where $\lambda_{i}$ are the eigenvalues of the self-adjoint compact operator $K$. This finally gives

$$
\begin{aligned}
\int_{\mathbb{T}^{2}} \rho(x) V(x) d x & \leq \sum_{i=1}^{n} \lambda_{i} \leq n^{1 / 2}\left(\sum_{i=1}^{n} \lambda_{i}^{2}\right)^{1 / 2} \leq \\
& \leq n^{1 / 2}\left(\operatorname{Tr} K^{2}\right)^{1 / 2} \leq \frac{n^{1 / 2} m^{-1}}{2 \sqrt{\pi}}\|V\|_{L^{2}}
\end{aligned}
$$

Setting $V(x):=\rho(x)$ we complete the proof of (5.3). 
Remark. In fact, we proved Proposition 5.3 in the scalar case, while $\theta_{j}$ in the proof of Theorem 3.1 are vector functions with mean value zero and $\operatorname{div} \theta_{j}=0$. The result with the same constant and the same proof still holds in this case, since on the torus the Helmgoltz-Leray projection commutes with the Laplacian and the orthonormal family of vector-valued eigenfunctions of the Stokes operator $v_{k}(x)=\frac{1}{2 \pi} \frac{k^{\perp}}{|k|} e^{i k x}, k \in \mathbb{Z}_{0}^{2}$ satisfy $\left|v_{k}(x)\right|=1 /(2 \pi)$ as in the scalar case (see (5.5)).

Remark. Inequality (5.5) is nothing more than a special case of the Kato-Seiler-Simon inequality $[29,13]$

$$
\|a(-i \nabla) b\|_{\varsigma_{q}} \leq(2 \pi)^{-d / q}\|a\|_{L^{q}}\|b\|_{L^{q}}
$$

on the torus with $d=2, q=2$ and $a(k)=1 /\left(|k|^{2}+m^{2}\right)$ (and with the same constant, since, as the next Lemma shows, $\left.\|a\|_{l^{2}\left(\mathbb{Z}_{0}^{2}\right)} \leq \sqrt{\pi} / m\right)$.

Lemma 5.4. For $m \geq 0$

$$
F(m):=m^{2} \sum_{k \in \mathbb{Z}_{0}^{2}} \frac{1}{\left(|k|^{2}+m^{2}\right)^{2}}<\pi .
$$

Proof. We assume that $m \geq 1$. We show below that (5.6) holds for $m \geq 1$, which proves the Lemma, since $F^{\prime}(m)>0$ on $m \in(0,1]$ and $F$ is increasing on $m \in[0,1]$.

We use the Poisson summation formula (see, e. g., [30])

$$
\sum_{m \in \mathbb{Z}^{n}} f(m / \mu)=(2 \pi)^{n / 2} \mu^{n} \sum_{m \in \mathbb{Z}^{n}} \widehat{f}(2 \pi m \mu),
$$

where $\mathcal{F}(f)(\xi)=\widehat{f}(\xi)=(2 \pi)^{-n / 2} \int_{\mathbb{R}^{n}} f(x) e^{-i \xi x} d x$. For the function $f(x)=1 /(1+$ $\left.|x|^{2}\right)^{-2}, x \in \mathbb{R}^{2}$, with $\int_{\mathbb{R}^{2}} f(x) d x=\pi$, this gives

$$
F(m)=\frac{1}{m^{2}} \sum_{k \in \mathbb{Z}^{2}} f(k / m)-\frac{1}{m^{2}} f(0)=\pi-\frac{1}{m^{2}}+2 \pi \sum_{k \in \mathbb{Z}_{0}^{2}} \widehat{f}(2 \pi m k) .
$$

Since $f$ is radial we have

$$
\widehat{f}(\xi)=\int_{0}^{\infty} \frac{J_{0}(|\xi| r) r d r}{\left(1+r^{2}\right)^{2}}=\frac{|\xi|}{2} K_{1}(|\xi|),
$$

where $K_{1}$ is the modified Bessel function of the second kind, and where the second equality is formula 13.51 (4) in [32].

Therefore we have to show that

$$
\sum_{k \in \mathbb{Z}_{0}^{2}} G(2 \pi m|k|)<\frac{1}{m^{2}}, \quad G(x)=\pi x K_{1}(x) .
$$


Next, we use the estimate [33]

$$
K_{1}(x)<\left(1+\frac{1}{2 x}\right) \sqrt{\frac{\pi}{2 x}} e^{-x}, x>0,
$$

which gives

$$
G(2 \pi m|k|)<\pi\left(\pi \sqrt{m|k|}+\frac{1}{4 \sqrt{m|k|}}\right) e^{-2 \pi m|k|} .
$$

For the first term we use that

$$
\sqrt{x} e^{-a x} \leq \frac{1}{\sqrt{2 e a}}
$$

with $a=\frac{1}{2} \pi m$ and $x=|k|$ (and keep three quarters of the negative exponent), while for the second term we just replace $1 / \sqrt{m|k|}$ by 1 , since $m \geq 1$ and $k \geq 1$. This gives

$$
G(2 \pi m|k|)<\pi\left(\sqrt{\frac{\pi}{e}} e^{-3 \pi m|k| / 2}+\frac{1}{4} e^{-2 \pi m|k|}\right) .
$$

Furthermore, we use that $|k| \geq \frac{1}{\sqrt{2}}\left(\left|k_{1}\right|+\left|k_{2}\right|\right)$ and, therefore,

$$
G(2 \pi m|k|)<\pi\left(\sqrt{\frac{\pi}{e}} e^{\frac{-3 \pi m\left(\left|k_{1}\right|+\left|k_{2}\right|\right)}{2 \sqrt{2}}}+\frac{1}{4} e^{-\sqrt{2} \pi m\left(\left|k_{1}\right|+\left|k_{2}\right|\right)}\right) .
$$

Thus, summing the geometric power series, we end up with

$$
\begin{array}{r}
F(m)<\pi-\frac{1}{m^{2}}+\pi \sqrt{\frac{\pi}{e}}\left(\frac{4}{\left(e^{\frac{3 \pi}{2 \sqrt{2}} m}-1\right)^{2}}+\frac{4}{e^{\frac{3 \pi}{2 \sqrt{2}} m}-1}\right)+ \\
+\frac{\pi}{4}\left(\frac{4}{\left(e^{\sqrt{2} \pi m}-1\right)^{2}}+\frac{4}{e^{\sqrt{2} \pi m}-1}\right) .
\end{array}
$$

Introducing the functions

$$
\varphi(x):=\frac{x^{2}}{e^{x}-1}, \quad \psi(x):=\frac{x}{e^{x}-1} .
$$

we have to show that

$$
\begin{aligned}
\Psi(m):= & 4 \sqrt{\frac{\pi}{e}}\left(\frac{2 \sqrt{2}}{3 \pi}\right)^{2}\left(\varphi\left(\frac{3 \pi}{2 \sqrt{2}} m\right)+\psi\left(\frac{3 \pi}{2 \sqrt{2}} m\right)^{2}\right)+ \\
& +\frac{1}{2 \pi^{2}}\left(\varphi(\sqrt{2} \pi m)+\psi(\sqrt{2} \pi m)^{2}\right)-\frac{1}{\pi}<0 .
\end{aligned}
$$

Note that the function $\psi$ is obviously decreasing for all $m \geq 0$, so all terms involving $\psi$ are decreasing. The function $\varphi(x)$ has a global maximum at

$$
x_{0}=1.5936 \ldots,
$$


and is decreasing when $x>x_{0}$. Since

$$
m_{1}:=\frac{2 \sqrt{2}}{3 \pi} x_{0}=0.47824<1, \quad m_{2}:=\frac{1}{\sqrt{2} \pi} x_{0}=0.35868<1,
$$

the function $\Psi(m)$ is decreasing for $m \geq 1$ and it is sufficient to verify the inequality for $m=1$ only. Since

$$
\Psi(1)=-0.141093 \cdots<0
$$

inequality (5.6) is proved.

Remark. Since $f(x)=1 /\left(|x|^{2}+1\right)^{2}$ is analytic, its Fourier transform decays exponentially and it follows from (5.8) that

$$
F(m)=\pi-\frac{1}{m^{2}}+O\left(e^{-\mathrm{C} m}\right) .
$$

This immediately gives that inequality (5.6) holds on $\left[m_{0}, \infty\right)$ and we have to somehow specify $m_{0}$ and then use numerical calculations to verify (5.6) on a finite interval $\left(0, m_{0}\right)$ (precisely this is the case of a similar estimates in [16]). Lemma 5.4 is one of the few examples when this can be done purely analytically. The key points are of course the explicit formula for the Fourier transform and uniform founds for $K_{1}$.

Acknowledgments. This work was done with financial support from the Moscow Centre of Fundamental and Applied Mathematics at the Keldysh Institute of Applied Mathematics (project 25-05-01).

\section{References}

[1] H. Araki, On an inequality of Lieb and Thirring. Lett. Math. Phys. 19:2, 167-170, (1990).

[2] A. Babin and M. Vishik, Attractors of Evolution Equations. Studies in Mathematics and its Applications, 25. North-Holland Publishing Co., Amsterdam, (1992).

[3] J. Bardina, J. Ferziger, and W. Reynolds, Improved subgrid scale models for large eddy simulation, in Proceedings of the 13th AIAA Conference on Fluid and Plasma Dynamics, (1980).

[4] Y. Cao, E. M. Lunasin, and E.S. Titi, Global well-posedness of the three-dimensional viscous and inviscid simplified Bardina turbulence models. Commun. Math. Sci. 4:4, 823-848, (2006).

[5] V. V. Chepyzhov and A. A. Ilyin, A note on the fractal dimension of attractors of dissipative dynamical systems. Nonlinear Anal. 44, 811-819, (2001).

[6] V. V. Chepyzhov and A. A. Ilyin, On the fractal dimension of invariant sets; applications to Navier-Stokes equations. Discrete Contin. Dyn. Syst. 10:1, 2, 117-135, (2004).

[7] V. V. Chepyzhov and M. I. Vishik, Attractors for Equations of Mathematical Physics. Amer. Math. Soc. Colloq. Publ. V.49. Providence, RI: Amer. Math. Soc., (2002). 
[8] V. V. Chepyzhov and M. I. Vishik, Trajectory attractors for dissipative 2D Euler and Navier-Stokes equations, Russian J. Math. Phys. 15, 156-170, (2008).

[9] V.V.Chepyzhov, A.A.Ilyin, S.V.Zelik. Vanishing viscosity limit for global attractors for the damped Navier-Stokes system with stress free boundary conditions. Physica D 376-377, 31-38, (2018).

[10] V. Chepyzhov, M. Vishik, and S. Zelik, Strong trajectory attractors for dissipative Euler equations. J. Math. Pures Appl. 96:4, 395-407, (2011).

[11] P. Constantin and C. Foias, Global Lyapunov exponents, Kaplan-Yorke formulas and the dimension of the attractors for the 2D Navier-Stokes equations. Comm. Pure Appl. Math. 38, 1-27, (1985).

[12] C. Foias, O. Manely, R. Rosa, and R. Temam, Navier-Stokes Equations and Turbulence. Cambridge Univ. Press, Cambridge, (2001).

[13] R. L. Frank, A. Laptev, and T. Weidl, Lieb-Thirring Inequalities, book in preparation.

[14] A. Haraux, Two remarks on dissipative hyperbolic problems. Nonlinear Partial Differential Equations and Their Applications, College de France Seminar, Vol. VII, H. Brezis, J.L. Lions (Eds.), Pitman, London. (1985).

[15] A. A. Ilyin, The Euler equations with dissipation, Sb. Math. 182:12, 1729-1739, (1991); English transl. in Math. USSR-Sb. 74:2 (1993).

[16] A. A. Ilyin, A. A. Laptev, and S. V. Zelik, Lieb-Thirring constant on the sphere and on the torus. J. Func. Anal. 279, 108784, (2020).

[17] A. A. Ilyin and E. S. Titi, Attractors to the two-dimensional Navier-Stokes- $\alpha$ models: an $\alpha$-dependence study. J. Dynam. Differential Equations 15, 751-778 (2003).

[18] A. A. Ilyin, A. Miranville, and E. S. Titi, Small viscosity sharp estimates for the global attractor of the 2-D damped-driven Navier-Stokes equations, Commun. Math. Sci. 2, 403-426, (2004).

[19] A. A. Ilyin, A. G. Kostianko and S. V. Zelik, Finite-dimensional attractors for damped Euler-Bardina model in three dimensions, in preparation.

[20] W. B. Jones and W. J. Thron, Continued Fractions. Analytic Theory and Applications. Wesley, London (1980).

[21] V. K. Kalantarov and E. S. Titi, Global attractors and determining modes for the 3D Navier-Stokes-Voight equations. Chin. Ann. Math. 30B:6 (2009), 697-714.

[22] O. A. Ladyzhenskaya, Attractors for Semigroups and Evolution Equations. Leizioni Lincei, Cambridge Univ. Press, Cambridge, (1991).

[23] E. H. Lieb, An $L^{p}$ bound for the Riesz and Bessel potentials of orthonormal functions. $J$. Func. Anal. 51, 159-165 (1983).

[24] E. Lieb, On characteristic exponents in turbulence. Comm. Math. Phys. 92, 473-480, (1984).

[25] V. X. Liu, A sharp lower bound for the Hausdorff dimension of the global attractors of the 2D Navier-Stokes equations. Comm. Math. Phys. 158, 327-339 (1993).

[26] L. D. Meshalkin and Ya. G. Sinai, Investigation of the stability of a stationary solution of a system of equations for the plane movement of an incompressible viscous liquid. Prikl. Mat. Mekh. 25, 1140-1143 (1961), English transl. in J. Appl. Math. Mech. 25 (1961). 
[27] J. Pedlosky, Geophysical Fluid Dynamics. Springer, New York, (1979).

[28] G. R. Sell and Y. You, Dynamics of Evolutionary Equations. Springer-Verlag, New York, (2002).

[29] B. Simon, Trace Ideals and Their Applications, 2nd ed. Amer. Math. Soc., Providence RI, (2005).

[30] E. M. Stein and G. Weiss, Introduction to Fourier analysis on Euclidean spaces. Princeton University Press, Princeton NJ (1972).

[31] R.Temam, Infinite Dimensional Dynamical Systems in Mechanics and Physics, 2nd ed. Springer-Verlag, New York (1997).

[32] G. N. Watson, A Treatise on the Theory of Bessel Functions, 2nd ed. Cambridge University Press, Cambridge (1995).

[33] Zhen-Hang Yang and Yu-Ming Chu, On approximating the modified Bessel function of the second kind. J. Ineq. and Appl. 41, 2-8 (2017).

[34] V. I. Yudovich, Example of the generation of a secondary stationary or periodic flow when there is loss of stability of the laminar flow of a viscous incompressible fluid. Prikl. Mat. Mekh. 29, 453-467 (1965), English transl. in J. Appl. Math. Mech. 29 (1965). 